\title{
Interactions and Collisions of Discrete Breathers in Two-Species Bose-Einstein Condensates in Optical Lattices
}

\author{
Russsell Campbell and Gian-Luca Oppo \\ Institute of Complex Systems, SUPA and Department of Physics, \\ University of Strathclyde, 107 Rottenrow, Glasgow G4 ONG, Scotland, UK.* \\ Mateusz Borkowski \\ Institute of Physics, Faculty of Physics, Astronomy and Informatics, \\ Nicolaus Copernicus University, Grudziadzka 5, 87-100 Torun, Poland
}

(Dated: November 26, 2014)

\begin{abstract}
The dynamics of static and traveling breathers in two-species Bose-Einstein condensates in a one-dimensional optical lattice is modelled within the tight-binding approximation. Two coupled discrete nonlinear Schrödinger equations describe the interaction of the condensates in two cases of relevance: a mixture of two ytterbium isotopes and a mixture of ${ }^{87} \mathrm{Rb}$ and ${ }^{41} \mathrm{~K}$. Depending on their initial separation, interaction between static breathers of different species can lead to the formation of symbiotic structures and transform one of the breathers from a static into a traveling one. Collisions between traveling and static discrete breathers composed of different species are separated in four distinct regimes ranging from totally elastic when the interspecies interaction is highly attractive to mutual destruction when the interaction is sufficiently large and repulsive. We provide an explanation of the collision features in terms of the interspecies coupling and the negative effective mass of the discrete breathers.
\end{abstract}

PACS numbers: 03.75.Mn, 03.75.Lm, 03.75.Kk, 05.45.Yv

\section{INTRODUCTION}

Bose-Einstein condensates have become a formidable tool for studying basic fundamentals of atomic physics [1, 2]. When confined to an optical lattice they serve as an interesting analogue to a solid-state system [3,4], providing means to study solid-state phenomena at an unprecedented level of parameter control. These include quantum phase transitions from the superfluid to Mott-insulator regimes [5], transport phenomena [6], Anderson localization [7], low-dimensional systems [8], discrete breathers [9] and solitons [10]. The latter can exist both with or without a periodic potential, although a lattice environment makes it possible for solitons and discrete breathers to exist even when the interactions in the BEC are repulsive ("gap solitons") [11-13]. Such states have been observed experimentally in [14]. Several other methods have been proposed in the literature, including the use of an additional harmonic potential [15] and boundary dissipations [16], which could also be used for non-demolition probing of these states [17].

The introduction of a second atomic species, i.e. the creation of a binary mixture of Bose-Einstein Condensates leads to even richer physics. In a harmonic trap the two species may be immiscible due to the interspecies interaction, leading to phase separation $[18,19]$. In an effectively one-dimensional environment, the repulsive interaction between atoms of different species ('interspecies' interactions) during the formation of the condensate can leave the mixture far from its ground state [20,21]. The interspecies interactions heavily influence the transport properties of a condensate in a lattice

\footnotetext{
*Electronic address: russell.campbell@strath.ac.uk
}

$[22,23]$. Repulsive interactions enable the formation and extend the stability region of the so-called symbiotic gap solitons [24-26], i.e. two-species solitons localized together in the same spot of the lattice. Somewhat counter-intuitively, an attractive interspecies interaction may split the two overlapping solitons [27].

So far most of the work has concentrated on the existence of two-species solitons and their stability [24-32]. Without a lattice potential, two-component (symbiotic) solitons were found with attractive interspecies interaction [33]. Collision of single species solitons without a lattice potential have also been shown to form symbiotic structures [34]. Collisions of symbiotic breathers were studied in [35]. In this paper we take a complementary approach - we start with well-defined single-species discrete solitons (two traveling breathers or a self-trapped state and a traveling breather, the first made of one species and the other of the second species) far apart in the lattice and simulate their collisions. For a single species BEC confined to a one-dimensional lattice collisions of traveling breathers have been analyzed in detail in [36]. Such an approach has also been used in the case of binary BECs in harmonic traps $[37,38]$. We perform our simulations using experimentally reachable conditions, with specific reference to two feasible experiments of two-species BECs in optical lattices.

The first of these experiments of interest has been performed in Kyoto where BECs of $\mathrm{Yb}$ atoms have been obtained separately with isotopes ${ }^{174} \mathrm{Yb}[39],{ }^{170} \mathrm{Yb}$ [40] and ${ }^{168} \mathrm{Yb}$ [41]. A stable mixture of the isotopes ${ }^{168} \mathrm{Yb}$ and ${ }^{174} \mathrm{Yb}$ was obtained [41], as well as a ${ }^{174} \mathrm{Yb}$ and ${ }^{176} \mathrm{Yb}$ mixture [42] which is unstable due to the negative scattering length of the ${ }^{176} \mathrm{Yb}$ component (see also [43]). The intra- and interspecies scattering lengths of ytterbium isotopes have been measured using data from one- [44] and two-color photoassociation spec- 
troscopy [45] and are now well established. The rich isotope structure of ytterbium enables mass tuning of the scattering length. It has been shown that, unlike in alkali-metal species, optical Feshbach resonances can be used to effectively change the intraspecies scattering length [46-48], thus raising hope for optical control of interactions between different isotopes, especially that now their positions are known from interisotope photoassociation spectroscopy [49]. Three bosonic isotopes of ytterbium, namely ${ }^{168,170,174} \mathrm{Yb}$, have positive intraspecies scattering lengths of the order of a few nanometers leading to similar stable condensates. Since the isotope shifts are small compared to the detuning of the far off resonant trap (FORT), the potential seen by different isotopes is basically identical. Consequently, given the mass ratios also close to one, this will result in very similar tunneling rates.

Even though these three isotopes are similar in terms of the single-species scattering length, the interspecies interactions of different pairs of isotopes differ dramatically. The interaction between a ${ }^{170} \mathrm{Yb}$ and ${ }^{174} \mathrm{Yb}$ atoms is described by a large negative scattering length of $-27.3 \mathrm{~nm}$, while for ${ }^{168} \mathrm{Yb}$ and ${ }^{170} \mathrm{Yb}$ it is positive and equal to $6.2 \mathrm{~nm}$. Halfway between these two is the case of ${ }^{168} \mathrm{Yb}$ and ${ }^{174} \mathrm{Yb}$ characterized by a negligible scattering length of $0.13(18) \mathrm{nm}$, where the two condensate species should essentially ignore each other.

An interesting mixture of heteronuclear BECs has been obtained in an experiment of Thalhammer et al. [50], where ${ }^{41} \mathrm{~K}$ and ${ }^{87} \mathrm{Rb}$ atoms are condensed together in an optical lattice. A remarkable property of this mixture is that the interspecies scattering length $a_{1,2}$ describing the effective interaction of colliding potassium and rubidium atoms can be tuned over a wide (both positive and negative) range using a magnetic Feshbach resonance, while the single-species scattering length remains positive for either species. This mixture is an example of one with a large tunneling rate ratio, in contrast to the ytterbium mixtures, where this ratio is close to one.

We present an analysis of the interaction and collisional behavior of discrete breathers in two-species BEC in optical lattices in the tight-binding approximation which has been successfully used to describe single-species experiments $[9,11,12,51]$. In Section II the derivation of the model and an estimate of the parameters are provided. Interaction of stationary breathers in close proximity to each other is described in section III. Collisions of traveling breathers and trapped states are described in Section IV as a function of the interspecies coupling parameter. Section V provides an explanation of the different kind of collisions observed in the numerical simulations where inelastic behavior is found in the mutually-repulsive case and elastic in the mutually-attractive case. Finally, the Appendix shows that the main results of the breather collisions and interactions are robust to survive in a model where the spatial variable is continuous rather than discrete.

\section{THE TIGHT-BINDING APPROXIMATION APPLIED TO A TWO-SPECIES BOSE GAS}

We analyze the behavior of the two-species Bose gas with the use of the tight-binding approximation, following the treatment described in [23]. The time-dependent Gross-Pitaevskii equations describing the dynamics of the two species' order parameters $\Psi_{i}$ (where $i=1,2$ ) read

$$
i \hbar \dot{\Psi}_{i}(\vec{r})=\left(-\frac{\hbar^{2}}{2 m_{i}} \nabla^{2}+U(\vec{r})+\sum_{j=1,2} g_{i, j}\left|\Psi_{j}(\vec{r})\right|^{2}\right) \Psi_{i}(\vec{r}),
$$

where the coefficients $g_{i, j}$ describe the effective mean-field intra- and interspecies interactions and are given by

$$
g_{i, j}=\frac{4 \pi \hbar^{2} a_{i, j}}{2 \mu_{i, j}}
$$

where $\mu_{i, j}=\left(m_{i}^{-1}+m_{j}^{-1}\right)^{-1}$ is the reduced mass of the atomic pair and $a_{i, j}$ is the scattering length relevant in the scattering properties of the species' atoms.

The external potential confining the BECs is due to two overlapping and counter-propagating laser beams that create a standing wave in the axial direction and, as a result, a periodic potential of depth $V_{0, i}$. The Gaussian profile of the two laser beams gives rise to an approximately harmonic off-axis confinement described by the frequencies $\omega_{r, i}$. Thus the external potential reads

$$
V_{i}=V_{0, i} \sin ^{2}(k x)+\frac{1}{2} m_{i} \omega_{r, i}^{2}\left(y^{2}+z^{2}\right)
$$

Note that real optical lattices also have a shallow harmonic potential superimposed in the axial direction, which we here assume to have negligible effects. We also introduce the lattice strengths $s_{i}=V_{0, i} / E_{R, i}$, where $E_{r, i}=\hbar^{2} k^{2} / 2 m_{i}$ is the recoil energy calculated for the lattice wavelength. The axial on-site frequency of the lattice is then $\omega_{i}=\sqrt{s_{i}} \hbar k^{2} / m_{i}$.

Both order parameters obey the normalization condition $\int \mathrm{d}^{3} \vec{r}\left|\Psi_{i}\right|^{2}=N_{i}$, where $N_{i}$ denotes the total number of atoms of the $i$-th species in the lattice.

If the lattice is strong enough, i.e. the trap depth is sufficiently large, the condensate is well localized around potential minima. For each of the mixture's order parameters we use the following ansatz [11, 23, 30]:

$$
\Psi_{i}=\sum_{n} \psi_{i, n}(t) \phi_{i}\left(\vec{r}-\vec{r}_{i, n}\right)
$$

where $\phi_{i}$ is an on-site wavefunction and $\vec{r}_{i, n}$ is the location of the $n$-th lattice site seen by the $i$-th species. When the atomic interactions are weak, the on-site ground state wavefunction can be replaced by the ground state harmonic oscillator wavefunction in the off-axis direction and a Wannier function [52] of the lowest band in the axial direction to account for tunneling. Consequently, $\left|\psi_{i, n}(t)\right|^{2}$ may be interpreted as the number of $i$-th species atoms in the $n$-th lattice site as a function of time and $\sum_{n}\left|\psi_{i, n}\right|^{2}=N_{i}$. 
Substituting this ansatz into the Gross-Pitaevskii equations and dropping all terms mixing different lattice sites except for the ones that describe tunneling (see $[11,23]$ ) one obtains

$$
\begin{aligned}
i \hbar \dot{\psi}_{i, n}= & -J_{i}\left(\psi_{i, n-1}+\psi_{i, n+1}\right) \\
& +\left(\lambda_{i, i}\left|\psi_{i, n}\right|^{2}+\lambda_{1,2}\left|\psi_{3-i, n}\right|^{2}+\epsilon_{i, n}\right) \psi_{i, n}
\end{aligned}
$$

where

$$
J_{i}=\int \mathrm{d}^{3} \vec{r} \phi_{i}\left(\vec{r}-\vec{r}_{i, n}\right)\left(\frac{-\hbar^{2}}{2 m_{i}} \nabla^{2}+V_{\mathrm{i}}\right) \phi_{i}\left(\vec{r}-\vec{r}_{i, n+1}\right)
$$

is the hopping integral describing the tunneling of the $i$ th species and is proportional to the intersite tunneling rate $\gamma_{i}=J_{i} / \hbar$, while $\epsilon_{i, n}=\int \mathrm{d}^{3} \vec{r} \phi_{i}\left(\vec{r}-\vec{r}_{i, n}\right)\left(\frac{-\hbar^{2}}{2 m_{i}} \nabla^{2}+V_{i}\right) \phi_{i}\left(\vec{r}-\vec{r}_{i, n}\right)$ is the on-site chemical potential. The self- and mutual interaction is described by the parameters $\lambda_{i, i}=g_{i, i} \int \mathrm{d}^{3} \vec{r}\left|\phi_{i}(\vec{r})\right|^{4}$ and $\lambda_{1,2}=g_{1,2} \int \mathrm{d}^{3} \vec{r}\left|\phi_{1}(\vec{r})\right|^{2}\left|\phi_{2}(\vec{r})\right|^{2}$, respectively.

\section{A. Normalization}

In order to move towards a more standard and computationally efficient form of two coupled discrete nonlinear Schrödinger equation (DNLSE), we introduce

$$
\begin{aligned}
z_{i, n} & =\sqrt{\frac{1}{N_{i}}} \psi_{i, n}^{*} \exp \left(-i \frac{\epsilon_{i} \tau}{\hbar \gamma_{1}}\right) \\
\tau & =\gamma_{1} t
\end{aligned}
$$

to obtain:

$$
\begin{aligned}
i \frac{d}{d \tau} z_{1, n} & =\Lambda_{1,1}\left|z_{1, n}\right|^{2} z_{1, n}+\Lambda_{1,2} \frac{N_{2}}{N_{1}}\left|z_{2, n}\right|^{2} z_{1, n} \\
& -z_{1, n-1}-z_{1, n+1} \\
i \frac{d}{d \tau} z_{2, n} & =\Lambda_{2,2}\left|z_{2, n}\right|^{2} z_{2, n}+\Lambda_{1,2}\left|z_{1, n}\right|^{2} z_{2, n} \\
& -\frac{\gamma_{2}}{\gamma_{1}}\left(z_{2, n-1}+z_{2, n+1}\right) .
\end{aligned}
$$

In Eqs. (9-10) we have defined the following parameters:

$$
\Lambda_{i, i}=\frac{\lambda_{i, i} N_{k}}{\hbar \gamma_{1}} \quad \Lambda_{1,2}=\frac{\lambda_{1,2} N_{1}}{\hbar \gamma_{1}}
$$

Thus the atomic distribution of each species over the entire lattice is normalized to unity:

$$
\sum_{n}\left|z_{i, n}\right|^{2}=1
$$

To ensure that the energy and density in the system are conserved, we use a symplectic fourth-order integrator of the Yoshida type $[53,54]$. The energy and density are both conserved up to 9 decimal places at each integration time step.
TABLE I: Values of parameters used in the simulations

\begin{tabular}{ccccccc}
\hline \hline Pair & $s_{1}$ & $s_{2}$ & $\gamma_{2} / \gamma_{1}$ & $\Lambda_{1,1}$ & $\Lambda_{2,2}$ & $\Lambda_{1,2}$ \\
\hline${ }^{168} \mathrm{Yb}+{ }^{170} \mathrm{Yb}$ & 3.19 & 3.27 & 0.96 & 5.28 & 1.368 & 2.51 \\
${ }^{170} \mathrm{Yb}+{ }^{174} \mathrm{Yb}$ & 3.27 & 3.43 & 0.91 & 1.368 & 2.486 & -12.24 \\
${ }^{87} \mathrm{Rb}+{ }^{41} \mathrm{~K}$ & 3.03 & 7 & 6.97 & 12.31 & 5.89 & (free) \\
\hline
\end{tabular}

\section{B. Estimate of the calculation parameters}

In Section III(A) we present results that model different Bose gas mixtures, notably mixtures of ytterbium isotopes and that of ${ }^{41} \mathrm{~K}+{ }^{87} \mathrm{Rb}$. At present, the only ytterbium isotope mixture BEC obtained so far is that of ${ }^{174} \mathrm{Yb}+{ }^{176} \mathrm{Yb}$ where, however, the ${ }^{176} \mathrm{Yb}$ part instantly collapses because of its negative scattering length [42]. Thus we focus on ytterbium mixtures of isotopes whose scattering length is positive, namely ${ }^{168} \mathrm{Yb},{ }^{170} \mathrm{Yb}$ and ${ }^{174} \mathrm{Yb}$. The latter two have already reached BEC separately $[39,40]$ while the major technical difficulty in reaching a ${ }^{168} \mathrm{Yb} \mathrm{BEC}$ is its low natural abundance of $0.13 \%$.

To calculate the self nonlinear parameter, $\lambda_{i, i}$, it is sufficient to approximate the on-site wavefunction $\phi_{i, n}(\vec{r})$ with a harmonic oscillator ground state, which is basically a Gaussian, to yield

$$
\lambda_{i, i}=a_{i, i} \sqrt{\frac{m \omega_{r}^{2} \omega}{2 \pi \hbar}}
$$

In the case of ytterbium mixtures the mutual interaction parameter $\lambda_{1,2}$ can also be estimated from the above formula as the masses of the two isotopes are very similar and the two wavefunctions are well overlapped.

In the case of the ${ }^{41} \mathrm{~K}+{ }^{87} \mathrm{Rb}$ mixture the two wavefunctions are differently shaped (one is narrower than the other due to the difference in masses). The difference in the masses of the two species has a further effect - the two clouds are separated due to gravity but an additional laser can be used to force overlap of the two species [55]. The scattering length of ${ }^{87} \mathrm{Rb}$ is $5.25 \mathrm{~nm}$ [56] while for ${ }^{41} \mathrm{~K}$ it is $3.1 \mathrm{~nm}[57,58]$. The interspecies scattering length can be changed by the use of a convenient magnetic Feshbach resonance [59] so that $\lambda_{1,2}$ can be considered as a free parameter.

To estimate the tunneling rates $\gamma_{i}$, it is not sufficient to approximate the on-site wavefunctions with Gaussians and one has to use the Wannier wavefunctions. This is because the tunneling rate is mostly determined by the wings of the on-site wavefunction which have an oscillatory-exponential rather than a Gaussian tail. In this case the tunneling rate can be approximated as [4]

$$
J_{i}=\hbar \gamma_{i}=\frac{4}{\sqrt{\pi}} E_{r, i} s_{i}^{3 / 4} \exp \left(-2 \sqrt{s_{i}}\right)
$$

The parameters for the ytterbium isotopes considered here are as follows. The lattice laser wavelength is $532 \mathrm{~nm}$; the lattice frequencies for both species are $\omega_{r}=2 \pi \times 100 \mathrm{~Hz}$ and $\omega=$ $2 \pi \times 15 \mathrm{kHz}$ which are close to the experimental realizations. For the sake of simplicity, we consider $N_{1}=N_{2}=O\left(10^{3}\right)$ as it is difficult to find initial conditions that would lead to a 
clear traveling breather at larger densities. This is a general property of gap solitons [15]. The results apply, however, to larger values of $N_{i}$ up to $10^{5}$.

In the case of the ${ }^{87} \mathrm{Rb}$ and ${ }^{41} \mathrm{~K}$ mixture we consider a lattice wavelength of $1064 \mathrm{~nm}$ and we take the (tunable) lattice depth to be $V_{e}=7 E_{r}$ for rubidium so that the same parameter for potassium is around 3. This is done to ensure that we can still use the tight-binding approximation (i.e. tunneling rates to further sites are at least an order of magnitude smaller than $\gamma_{i}$, see table I in [4]). On the other hand the tunneling rate needs to be large enough for traveling breathers to exist.

The calculation parameters discussed above are shown in table I.

\section{BREATHER INTERACTION}

It has been demonstrated that initially Gaussian wavepackets can evolve via the single species DNLSE into static breathers $[9,11,51]$. If the wavepacket is given a momentum in a certain direction, traveling breathers that translate across the lattice can also be formed. The general expression of the initial wavepacket is:

$$
z_{i, n}=\sqrt{\frac{1}{\sqrt{2 \pi \sigma_{i}^{2}}}} \exp \left(-\frac{\left(n-\bar{n}_{i}\right)^{2}}{4 \sigma_{i}^{2}}\right) e^{i p_{i} n}
$$

where $\sigma_{i}$ is the initial width of the Gaussian cloud, and $\bar{n}_{i}$ is its position. For the single species case, low nonlinearity and values of $\left|p_{i}\right|$ between zero and $\pi / 2$, corresponding to a positive $\cos p_{i}$, the cloud expands diffusively within the lattice. Localization into static breathers is then observed when increasing the repulsive self-interaction $\Lambda_{i, i}$. However, when the pseudomomentum crosses $\pi / 2$ and the repulsive self-interaction $\Lambda_{i, i}$ is not too large, traveling breathers are formed (unless $\cos p_{i}$ is exactly 1 , in which case the breather is stationary) $[9,11,16,17,51,60]$.

In this section, we run simulations starting from stationary breathers of ${ }^{168} \mathrm{Yb}$ and ${ }^{170} \mathrm{Yb}$ in separate positions. These are formed by running simulations of initially Gaussian wavepackets with $\Lambda_{1,2}=0$ and letting them reshape with dissipation applied at the boundaries to get rid of the background (see [9] for a detailed description of the effects of dissipation). The final stable single-species breathers are of the "staggered" type and evolve in time as $z_{i, n}(\tau)=$ $\exp \left(-i \mu_{i} \tau\right)(-1)^{n} v_{i, n}$ where $v_{i, n}$ satisfies the stationary equations

$$
\begin{array}{r}
\mu_{1} v_{1, n}-\left(v_{1, n+1}+v_{1, n-1}\right)-\left(v_{1, n}^{2}+\beta v_{2, n}^{2}\right) v_{1, n}=0 \\
\mu_{2} v_{2, n}-\frac{\gamma_{2}}{\gamma_{1}}\left(v_{2, n+1}+v_{2, n-1}\right)-\left(\alpha v_{2, n}^{2}+\beta v_{1, n}^{2}\right) v_{2, n}=0
\end{array}
$$

with $\beta=\Lambda_{1,2} / \Lambda_{1,1}$ and $\alpha=\Lambda_{2,2} / \Lambda_{1,1}$. The breathers are spatially localized (see inset of Fig. 1 (a), in which the exponential tails of the breathers are seen clearly in a logarithmic scale). Substituting the exponential ansatz

$$
v_{i, n}=A_{i} \exp \left(-q_{i}|n|\right)
$$

into equations (16), as in [26], we find that the decay rates $q_{i}$ and frequencies $\mu_{i}$ of the breathers are related via:

$$
\begin{array}{r}
q_{1}=\ln \left(\mu_{1}+\sqrt{\mu_{1}^{2}-4}\right) \\
q_{2}=\ln \left(\frac{\gamma_{2}}{\gamma_{1}} \mu_{2}+\sqrt{\left(\frac{\gamma_{2}}{\gamma_{1}} \mu_{2}\right)^{2}-4}\right) .
\end{array}
$$

Following again [26], we obtain variational equations from an effective Lagrangian given by

$$
\begin{aligned}
& A_{1}^{2} \operatorname{coth}\left(2 q_{1}\right)+\beta A_{2}^{2} \operatorname{coth}\left(q_{1}+q_{2}\right)=2 \tanh \left(q_{1} / 2\right)+ \\
& \quad\left(\mu_{1}-2\right) \operatorname{coth}\left(q_{1}\right) \\
& \alpha A_{2}^{2} \operatorname{coth}\left(2 q_{2}\right)+\beta A_{1}^{2} \operatorname{coth}\left(q_{1}+q_{2}\right)=\frac{2 \gamma_{2}}{\gamma_{1}} \tanh \left(q_{2} / 2\right)+ \\
& \quad\left(\mu_{2}-\frac{2 \gamma_{2}}{\gamma_{1}}\right) \operatorname{coth}\left(q_{2}\right)
\end{aligned}
$$

Solutions with both $A_{1}$ and $A_{2}$ different from zero and corresponding to symbiotic breathers may exist for $\beta>0$. In contrast to [26], single-species staggered breathers exist in the uncoupled case of $\beta=0$. For this reason we cannot exclude the existence of symbiotic breathers for $\beta<0$. We can however state that symbiotic breathers that are more localized than the original single-species interacting breathers, such as those generated in the numerical simulations below, do not exist for $\beta<0$. In the case of increasing values of $q_{i}$, one can see from Eqs. (19) that the fastest growing terms are those containing $\mu_{i}$ that depends exponentially on $q_{i}$, while the intensities $A_{i}^{2}$ grow only linearly with $q_{i}$. Such growth can only be compensated by a positive term containing $\operatorname{coth}\left(q_{1}+q_{2}\right)$ on the left hand sides of Eqs. (19) and corresponding to $\beta>0$. We can then conclude that no symbiotic breathers corresponding to further localization of the atomic density can form with negative $\beta$ from the interaction of two staggered single-species breathers.

In the simulations presented in this subsection, the frequencies of the single-species breathers for $\Lambda_{1,2}=\beta=0$ are found to be $\mu_{1}=2.1$ and $\mu_{2}=4.1$. The decay rates are then calculated using equations (18) to get $q_{1}=0.3$ and $q_{2}=1.3$, respectively. Once the breathers are formed and the background noise has vanished, we turned the dissipations off and measured values of $q_{1}$ and $q_{2}$ within a few percents from the predictions of (18).

To study the interactions of these breathers, we have set $\Lambda_{1,2}$ to the value of 2.51 and changed the initial distance $D$ between the centers of two single-species breathers. With the stationary breathers centered on the same site at the start of the simulation $(D=0)$, a symbiotic staggered breather is formed, with the ${ }^{170} \mathrm{Yb}$ breather moving atomic density into the background (see the insets of Fig. 1 (a) and (b) where we show that the background of the ${ }^{170} \mathrm{Yb}$ breather has increased from around $10^{-6}$ to around $10^{-2}$ ). The density profile of the symbiotic breather is then different from that of the two single-species breathers corresponding to $\Lambda_{1,2}=0$. The presence of the interspecies interaction increases both 

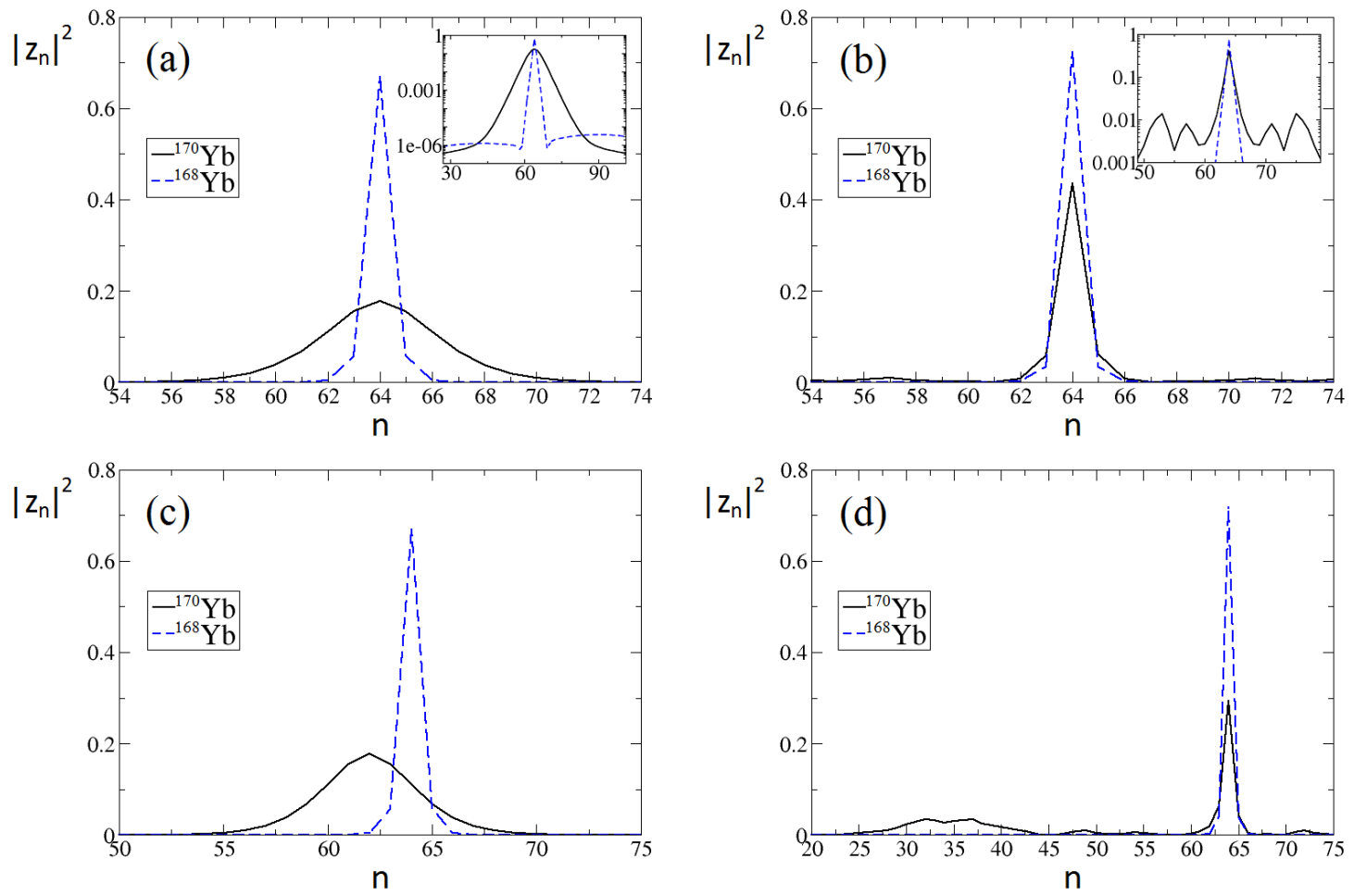

FIG. 1: (Color online) Density profiles of ${ }^{170} \mathrm{Yb}$ (black solid line) $+{ }^{168} \mathrm{Yb}$ (blue dashed line) mixture. (a) Initial density profile of ${ }^{170} \mathrm{Yb}+$ ${ }^{168} \mathrm{Yb}$ breathers with $D=0$. Inset displays the logarithmic profile of this, which shows the exponential tails of the breathers. (b) Density profile of ${ }^{170} \mathrm{Yb}+{ }^{168} \mathrm{Yb}$ breathers at $\tau=1000$ after $\Lambda_{1,2}$ is switched on for $D=0$. Note that the density profiles have changed when forming the symbiotic breather. The inset shows the logarithmic profile, in which the background of the ${ }^{170} \mathrm{Yb}$ species can be seen more clearly. (c) Initial density profile of ${ }^{170} \mathrm{Yb}+{ }^{168} \mathrm{Yb}$ breathers with $D=2$. The wavefunctions of the two species still overlap significantly. (d) Density profile of ${ }^{170} \mathrm{Yb}+{ }^{168} \mathrm{Yb}$ breathers at $\tau=1000$ after $\Lambda_{1,2}$ is switched on for $D=2$. Note that the ${ }^{170} \mathrm{Yb}$ is smaller and some of the background has become localized to the left of the main breather.

the frequencies $\mu_{i}$ and the exponential slopes $q_{i}$ of the breathers, making the breathers narrower and more localized. The frequencies are increased to $\mu_{1}=3.1$ and $\mu_{2}=5.3$ and the decay rates are both increased to $q_{1}=1.0$ and $q_{2}=1.6$. The measured values of $\mu_{i}, q_{i}$ and $A_{i}$ satisfy the variational Eqs. (19) within less than one percent in spite of the approximations made.

This behaviour keeps occurring when the initial distance between the breathers is larger, but still small enough that the initial density profiles overlap significantly. An example of this behavior is shown in Fig. 1 (c) and (d), for $D=2$. Here, we see that less of the ${ }^{170} \mathrm{Yb}$ atoms join with the symbiotic breather and more are expelled into the background. A small traveling packet is then formed from the atoms in the background. The reshaping process of the ${ }^{168} \mathrm{Yb}$ breather is much the same as with $D=0$. The formation and evolution of a traveling breather out of the interaction of two static breathers of separate species is presented in Fig. 2. With $D=8$, the initial density profiles of the breathers only overlap at the tails (see Fig. 2(b)). The result of this is that only a small density of ${ }^{170} \mathrm{Yb}$ atoms contributes to the symbiotic breather. As $D$ is increased, more ${ }^{170} \mathbf{Y b}$ atoms go into the backeground to support the traveling breather and less in the symbiotic one. A nonzero background is required for the traveling breather to exist [9]. The inset of Fig. 2(b) shows the logarithmic profile of the ${ }^{170} \mathrm{Yb}$ condensate, in which it is clear that the background is significantly higher than that of the initial condition.

It is important to stress that the motion of the traveling breather made of ${ }^{170} \mathrm{Yb}$ atoms is due to the interaction of the two species via $\Lambda_{1,2}$ being different from zero. In the case of no interaction $\left(\Lambda_{1,2}=0\right)$ both breathers remain stationary at all times. No symbiotic breather has been observed to form via the interaction of two single-species breathers for $\Lambda_{1,2}<0$.

\section{COLLISION OF TRAVELING AND STATIONARY BREATHERS}

Having assessed the interaction of discrete breathers of different atomic species when set in close proximity, we investigate here the collision of these breathers having set one or both of them in motion by using the pseudomomentum $p_{i}$ in the initial conditions given by (15). Experimentally, traveling breathers can be constructed by accelerating the lattice confin- 
(a)

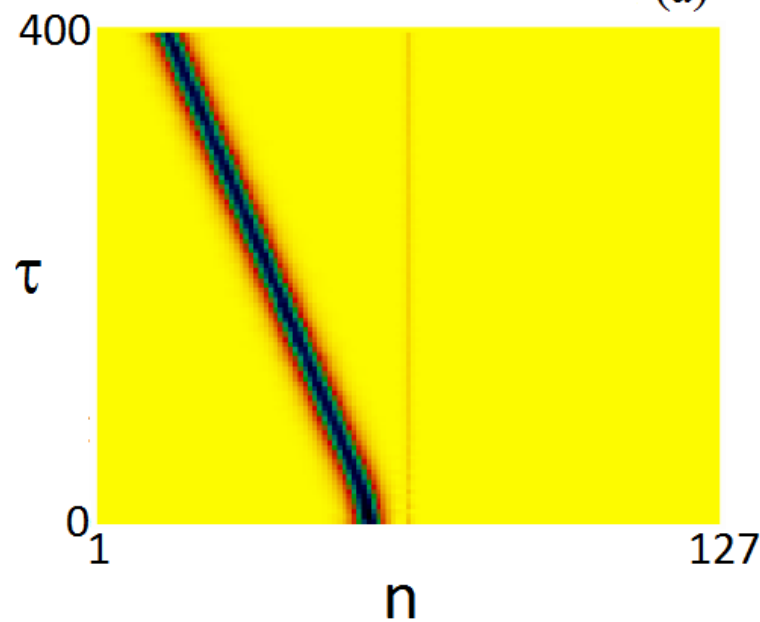

(b)

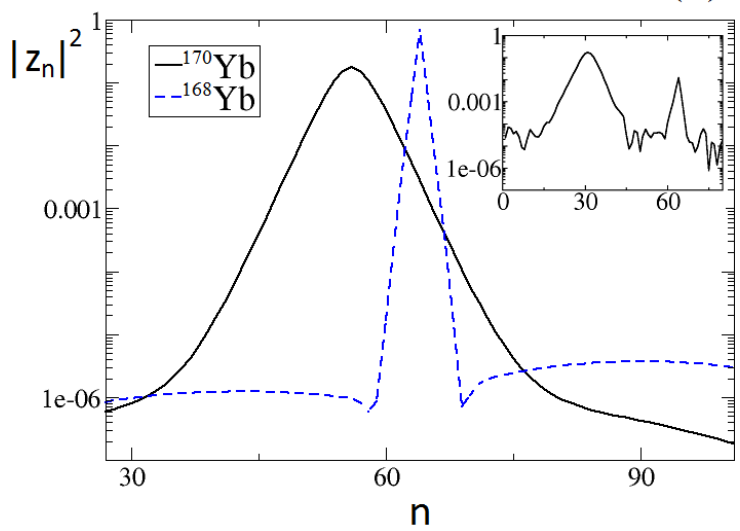

FIG. 2: (Color online) Interaction between initially stationary breathers in the ${ }^{170} \mathrm{Yb}$ (black solid line) $+{ }^{168} \mathrm{Yb}$ (blue dashed line) mixture for $D=8$. (a) Evolution of ${ }^{170} \mathrm{Yb}$ part of the mixture. The majority of ${ }^{170} \mathrm{Yb}$ atoms forms a traveling breather while the remaining atomic density is absorbed by the ${ }^{168} \mathrm{Yb}$ breather to form a symbiotic breather. We only show the ${ }^{170} \mathrm{Yb}$ part of the mixture since the ${ }^{168} \mathrm{Yb}$ evolution is rather straightforward, with the breather highly localized in the center. (b) Density profile of the initial condition in a logarithmic scale, showing the overlap at the tails. The inset shows the density profile of the ${ }^{170} \mathrm{Yb}$ mixture at $\tau=1000$. Note that the background is significantly higher than in the iniital condition, allowing the breather to travel.

ing the condensate. Such accelerations were realized experimentally in [61] and were used to observe a lensing effect on the condensate cloud. In the case of two different species in the same lattice the difference in the masses will naturally lead to different pseudomomenta of the clouds after acceleration to the same group velocity, thus making collisions possible.

In Fig. 3, we show three symplectic simulations of two colliding traveling breathers, with carefully chosen parameters so that there would be a minimal amount of sound-waves emitted from the initial Gaussian distributions. In all simulations presented in Fig. 3 the breathers start from the same initial conditions but with different values of the interspecies interaction (a)

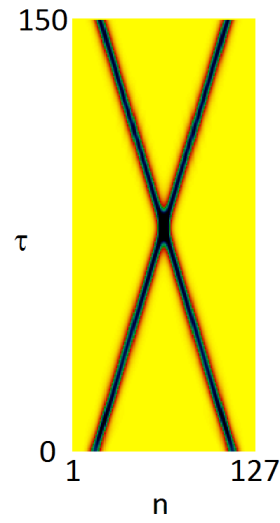

(b)

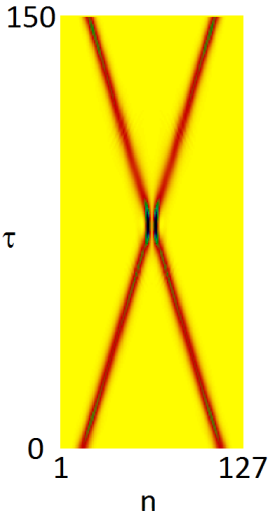

(c)

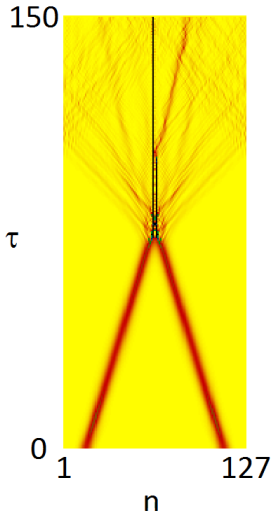

FIG. 3: (Color online) Collision of two "clean" traveling breathers, with minimal sound-waves. The initial conditions are $\Lambda_{1}=\Lambda_{2}=$ $1.1, \bar{n}_{1}=16, \bar{n}_{2}=112, \sigma_{1}=\sigma_{2}=3$ and $\cos p_{1}=\cos p_{2}=-0.95$ for all panels. Note that $p_{1}=-p_{2}$ and therefore the traveling breathers move in opposite directions. $\Lambda_{1,2}=0$ is set to: (a)0, (b)-20 and (c)30. In (a), the breathers ignore each other acting as if the other species was not present. In (b), the breathers collide elastically. In (c), the breathers are destroyed and a new symbiotic breather is created. The color here represents the total density of the two species, unlike the other figures.

parameter $\Lambda_{1,2}$. The color represents the (normalized) atomic density $\left|z_{n, i}\right|^{2}$ with $i=1$ for one species and $i=2$ for the other. The simulations start with two Gaussian wavepackets of the form (15) with $\cos p_{1}=\cos p_{2}=-0.95$ and $p_{1}=-p_{2}$ to form two colliding traveling breathers.

In Fig. 3(a) when the interspecies interaction parameter $\Lambda_{1,2}$ is set to zero, the breathers follow the dynamics of singlespecies condensates and pass through each other unaffected. When $\Lambda_{1,2}$ is a non-zero value, the two species affect each other when occupying the same lattice sites. This is shown in Fig. 3(b) and (c). At the beginning of the simulations, when the breathers are far apart from one another in the lattice, they follow the same path as in Fig. 3(a), until they collide. For large negative values of $\Lambda_{1,2}$, the breathers collide elastically, as shown in figure $3(\mathrm{~b})$. In this example, $\Lambda_{1,2}=-20$ and the breathers become narrower when they collide. In Fig. 3(c), $\Lambda_{1,2}$ is changed to a positive value and the collision is not elastic. At the collision, the breathers explode, emitting a large amount of sound waves and a stationary symbiotic soliton composed of both species is formed.

It is worth noting that elastic collision occurs when the interspecies interaction parameter is negative, which would normally imply attractive interactions between the two species. Normally the two clouds try to achieve maximal overlap in order to minimize energy [34], while in our case the tendency is to minimize the overlap and retain separation of at least a few lattice sites. An explanation of this phenomenon by using the negative effective mass of the discrete breathers is provided in Section V.

It should be noted that the single-species traveling breathers are not exact solutions of the DNLS equation due to the emission of sound waves [62]. Nevertheless, they 
(a): $\tau$

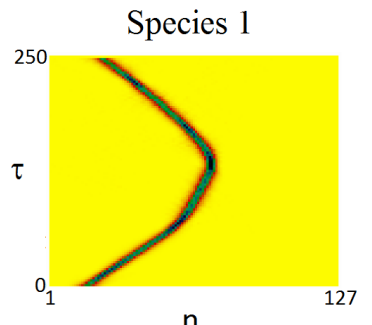

$\mathrm{n}$

(b): $\tau$

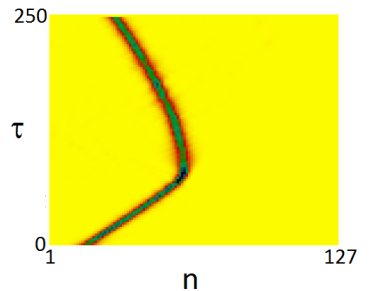

Species 2

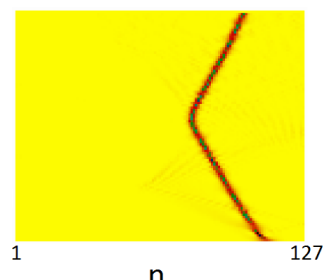

n

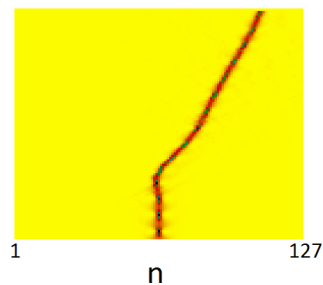

(a): $\tau$

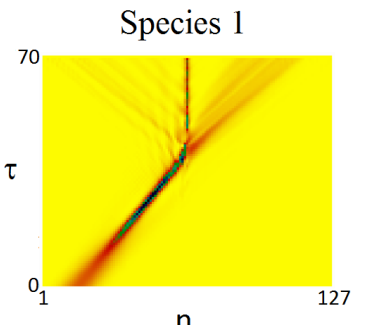

$\mathrm{n}$

(b): $\tau$

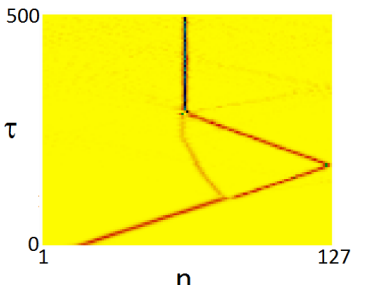

Species 2
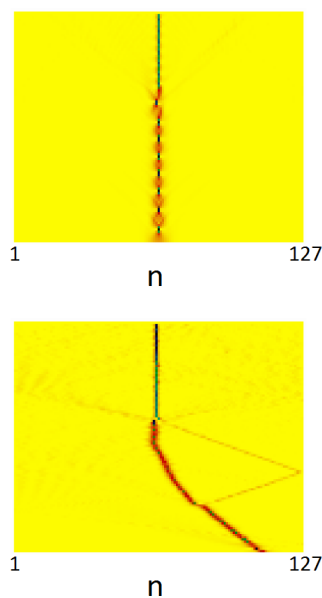

FIG. 4: (Color online) Collisions of two breathers in the ${ }^{170} \mathrm{Yb}$ (Species 1) $+{ }^{174} \mathrm{Yb}$ (Species 2) mixture characterized by a large negative interspecies scattering length of $-27.3 \mathrm{~nm}$. The Gaussian parameters for the initial condition in (a) are $\bar{n}_{1}=16, \bar{n}_{2}=112$, $\sigma_{1}=\sigma_{2}=3$ and $\cos p_{1}=\cos p_{2}=-0.95$. For (b), the physical situation is the same as in Fig. 4 (a), except that $\bar{n}_{2}=64$ and $\cos p_{2}=-1$ to make a stationary breather. In (a), 2 traveling breathers collide elastically. In (b).A traveling breather transfers large part of its (pseudo)momentum to a stationary one and nearly stops.

survive well localized for extremely long timescales. For the simulations in Fig. 3, we have carefully chosen the parameters so that the amount of sound-waves emitted from the breathers is minimal and find that, at the moment of collision, the breathers have only lost less than $0.47 \%$ of their densities. We also found that without interactions between species, they survive in simulations with timescales of $\tau \approx 10^{6}$, which is much higher than the collision times considered here.

In the following we turn our attention to values of the parameters chosen from table I to model mixtures of ytterbium isotopes and that of ${ }^{41} \mathrm{~K}+{ }^{87} \mathrm{Rb}$ in realistic configurations. In these simulations, although the initial condition emits large amount of noise, we show that the main dependance of the collision from the interaction parameter $\Lambda_{1,2}$ remains that displayed in Fig. 3.

In Fig. 4 we show numerical simulations of two colliding breathers in the ${ }^{170} \mathrm{Yb}+{ }^{174} \mathrm{Yb}$ mixture that display similar results to that of Fig. 3(b). Note that for all following simulations, the dynamics of each species is shown in separate panels, unlike Fig. 3. For example, the left column in Fig. 4 shows the ${ }^{170} \mathrm{Yb}$ species, while the right column shows the ${ }^{174} \mathrm{Yb}$ species. In Fig. 4(a), at about $\tau \approx 90$ the two breathers collide elastically, as in Fig. 3(b) since the interspecies scattering length is large and negative.

Figure 4(b) shows a similar situation, except that now one of the breathers $\left({ }^{174} \mathrm{Yb}\right)$ is at first stationary $\left(\cos p_{2}=-1\right)$. After the collision the initially traveling breather (almost) stops while the other, up to now stationary, starts traveling. One
FIG. 5: (Color online) Inelastic collisions of ytterbium isotopes. In (a), a traveling $\left({ }^{170} \mathrm{Yb}\right.$, species 1$)$ and stationary breather $\left({ }^{168} \mathrm{Yb}\right.$, species 2) collide for a positive interspecies scattering length of $6.2 \mathrm{~nm}$. The initial condition parameters are $\bar{n}_{1}=16, \bar{n}_{2}=64$, $\sigma_{1}=5, \sigma_{2}=3, \cos p_{1}=0.8, \cos p_{2}=1.0$. As in Fig. 3(c), the traveling breather is destroyed and a new symbiotic soliton is created. In (b) we have the same physical situation as in Fig. 4 (a), except that the parameter $\Lambda_{1,2}$ describing the interspecies has now been increased to 4 , corresponding to a positive interspecies scattering length of $8.9 \mathrm{~nm}$. In this regime, the two breathers tunnel through each other. Note that a small part of each breather is trapped inside the other forming double-species traveling breathers.

could argue that this is a manifestation of a form of conservation of momentum. Again, as in Fig. 3, the elastic behaviour occurs even though the ${ }^{170} \mathrm{Yb}$ and ${ }^{174} \mathrm{Yb}$ pair is described by a large negative scattering length of $a_{1,2}=-27.3 \mathrm{~nm}$, which, under normal circumstances, stands for attraction between the atoms of the two species.

Compared to the examples in Fig. 3, there is a much larger amount of sound waves emitted from the breathers in Fig. 4 due to the parameter values used from Table I. For example, in Fig. 4(a), the ${ }^{170} \mathbf{Y b}\left({ }^{174} \mathbf{Y b}\right)$ breather loses $1.83 \%(15.5 \%)$ of its density from the sound waves. Small amplitude sound waves do not affect the main collision in a significant way since the central part of each breather acts as an effective barrier to the sound waves emitted by the breather of the other species. Sound waves that would normally expand over the entire lattice are now confined to a region limited by the central parts of the two breathers. The scattering of sound waves due to discrete breathers is investigated in detail in [62].

A qualitatively different behavior from the above and similar to that in Fig. 3(c), is found in the case of the ${ }^{168} \mathrm{Yb}+{ }^{170} \mathrm{Yb}$ pair described by a positive (repulsive) scattering length of $a_{1,2}=+6.2 \mathrm{~nm}$. Due to the large intraspecies interaction of ${ }^{168} \mathrm{Yb}$ it is difficult to construct a clear traveling breather and therefore we limit ourselves to the case where the ${ }^{168} \mathrm{Yb}$ breather is initially stationary. This situation is presented in Fig. 5(a). As in Fig. 3(c), at the time of impact $(\tau \approx 40)$ 
(a): $\tau$

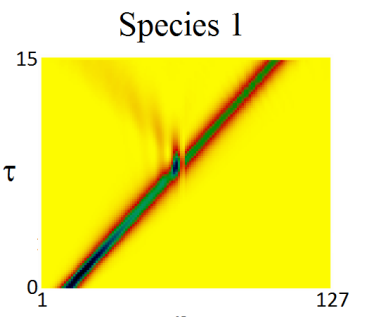

n

(b): $\tau$

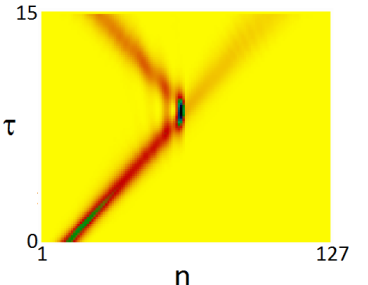

Species 2
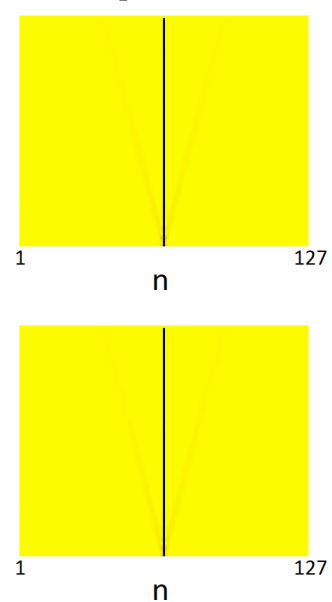

FIG. 6: (Color online) A collision of a traveling $\left({ }^{41} \mathrm{~K}\right.$, species 1) and a stationary breather $\left({ }^{87} \mathrm{Rb}\right.$, species 2$)$, with the interspecies interaction parameter $\Lambda_{1,2}=3$ (a) and $\Lambda_{1,2}=-9$ (b). The initial condition parameters are $\bar{n}_{1}=64, \bar{n}_{2}=12, \sigma_{1}=0.5, \sigma_{2}=3$, $\cos p_{1}=1, \cos p_{2}=-0.9$. In (a), the traveling breather tunnels almost completely through the self-trapped state, while in (b), the traveling breather bounces elastically from the self-trapped state with only a minor proportion tunneling through.

the two breathers literally explode emitting a large amounts of sound waves and forming a double-species symbiotic stationary breather. In this state, the two component wavefunctions are well overlapped. Moreover, the final breather is much narrower than any of the original breathers before the collision. The frequency of oscillation of the two final co-located and co-existing breathers is species dependent. In the case displayed in Fig. 5(a) the frequency of the ${ }^{168} \mathrm{Yb}$ breather is about 1.5 times that of the ${ }^{170} \mathrm{Yb}$ breather located on the same site.

To further explore these phenomena we carried out a simulation for the physical situation described in Fig. 4(a) of the ${ }^{170} \mathrm{Yb}+{ }^{174} \mathrm{Yb}$ mixture but with the interaction parameter $\Lambda_{1,2}$ increased to 4 corresponding to an interspecies scattering length of $a_{1,2}=8.9 \mathrm{~nm}$. Such an increase can potentially be achieved using an optical Feshbach resonance. In this situation, we observe the collision of two traveling breathers with a positive interspecies interaction as shown in Fig. 5(b). We find yet another collision behavior with these parameters: the two breathers mainly tunnel through each other but at each collision a fraction of the atomic species in one soliton becomes trapped inside the other. Moreover, the breathers appear to accelerate or decelerate for a brief time during the strong interaction. In this simulation we consider that lattice sites outside the condensate are empty resulting in traveling breathers to "bounce" off at reflective boundaries [63]. This is realized experimentally by fixing the size of the condensate with external magnetic fields. We have included this effect here to show that when the breathers collide with each other for a second time, the result is a stationary symbiotic breather.

Quite different results are obtained for the ${ }^{41} \mathrm{~K}+{ }^{87} \mathrm{Rb}$ mix-

ture, characterized by a large tunneling rate ratio of $\gamma_{2} / \gamma_{1} \approx$ 6.97. This, together with the large self-interaction of $\mathrm{Rb}$, changes the physics dramatically.

In Fig. 6 we show a collision of a traveling breather and a self-trapped state of rubidium atoms. Within the range of our simulation parameters that simulates possible experimental realizations, it has proved not possible to initiate a traveling breather state with the $\mathrm{Rb}$ condensate due to its large self-interaction. We set the interspecies interaction parameter $\Lambda_{1,2}=3$ for Fig. 6(a) and $\Lambda_{1,2}=-9$ for Fig. 6(b). In both cases the rubidium breather acts only as a potential barrier, through which some of the incoming potassium soliton can either reflect or tunnel. This behavior, which contrasts with the phenomena seen in the simulation with ytterbium, can be attributed to the drastically different tunneling rates of potassium and rubidium.

\section{THE COLLISION MECHANISM}

To understand the mechanisms at the base of the collision outcomes, we have investigated the dependence of the result of the collisional process with respect to the heteronuclear interaction. By treating the discrete condensate wavefunctions as distributions we define the mean lattice site

$$
\left\langle n_{i}\right\rangle=\sum_{n} n\left|z_{i, n}\right|^{2}
$$

and its standard deviation,

$$
\left\langle\Delta n_{i}\right\rangle=\left(\sum_{n}\left(n-\left\langle n_{i}\right\rangle\right)^{2}\left|z_{i, n}\right|^{2}\right)^{1 / 2} .
$$

These two parameters describe the global behavior of the condensate. In fact, if a breather splits or is destroyed in a collision, the standard deviation increases dramatically. To assess the local behavior, i.e. looking for a new breather created in a collision, we also search for the site with the largest number of atoms, $n_{\max , i}$ and attempt to estimate the new breather's width (Full Width Half Maximum, FWHM) by counting the adjacent sites which contain at least half the number of atoms of those in the site of the maximum.

Figure 7 shows the parameters $\left\langle n_{i}\right\rangle, n_{\max , i},\left\langle\Delta n_{i}\right\rangle$ and the FWHM as a function of the mutual interaction parameter $\Lambda_{1,2}$. For each value of $\Lambda_{1,2}$ a simulation was performed up to $\tau=150$, just past the collision. The initial conditions and interaction parameters are the same as in Fig.4, except for the scanned $\Lambda_{1,2}$ and the tunneling ratio $\gamma_{2} / \gamma_{1}$, which is set to 1 .

Four different regimes can be identified from Fig. 7. On the left, for $\Lambda_{1,2}$ lower than about -2.0 , two traveling breathers collide elastically and remain basically unaffected by the collision. This is the situation shown in figure 4. Then, there is a transition point at $\Lambda_{1,2} \approx-1.5$ where the each breather splits into two as they collide. This results in a sudden increase of $\langle\Delta n\rangle$ for each species. Note that this increase differs between the two species; for ${ }^{174} \mathrm{Yb}$ it's maximum is located at $\Lambda_{1,2} \approx-2$ as opposed to $\Lambda_{1,2} \approx-1$ for ${ }^{170} \mathrm{Yb}$, quite probably as a result of the different self-interaction parameters. 

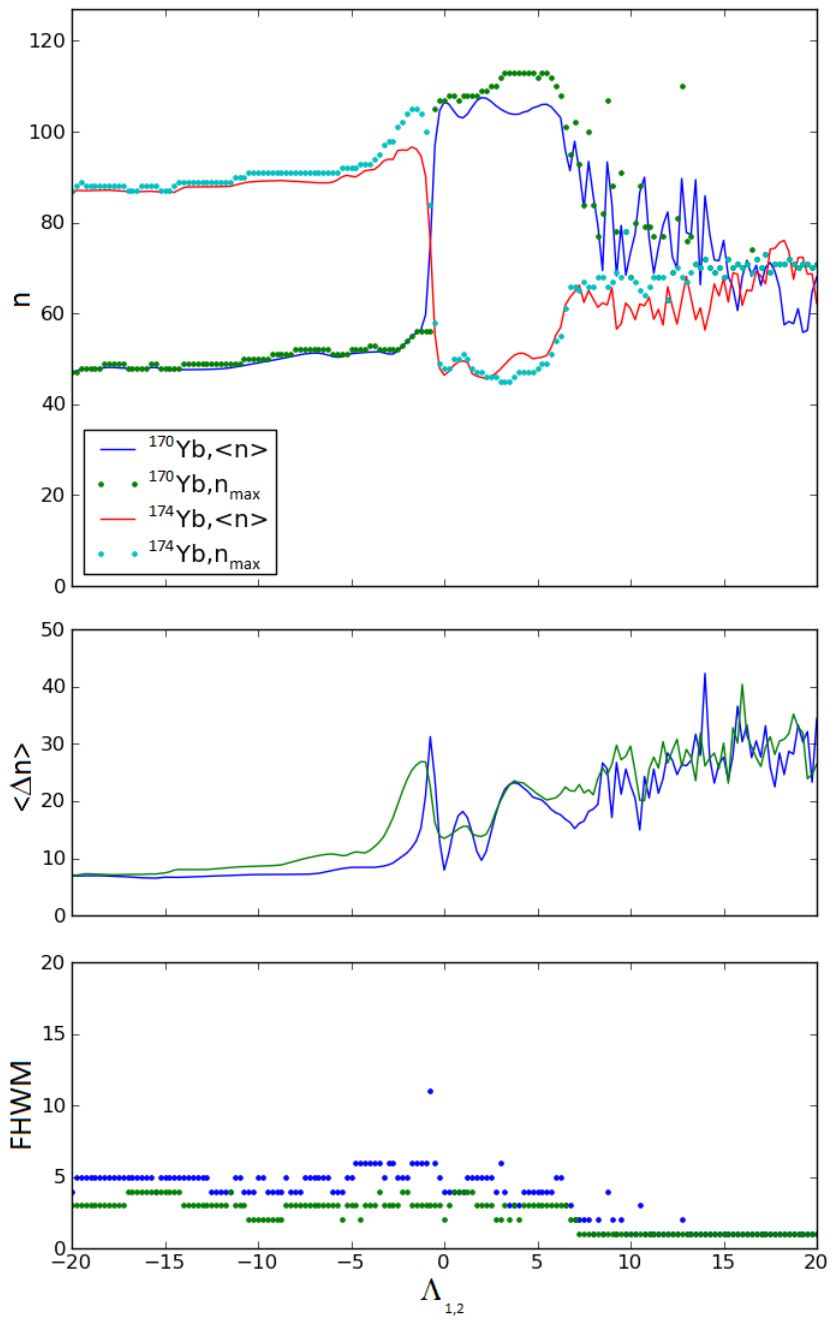

FIG. 7: (Color online) The collision outcome as a function of the interspecies interaction, $\Lambda_{1,2}$. The top panel displays the mean and the peak site per species, the center panel the standard deviation per species, and the bottom panel the FWHM per species as defined in the text. Symplectic simulations corresponding to the ${ }^{170} \mathrm{Yb}+{ }^{174} \mathrm{Yb}$ mixture.

For small, but positive values of $\Lambda_{1,2}$ (i.e. less than 6) the two breathers tunnel through each other. Note that $\langle\Delta n\rangle$ remains low in this regime (about 15 sites, growing slowly to 20) which means that the breathers are not destroyed. This is shown in Fig. 5(b) for $\Lambda_{1,2}=4$. As the two breathers tunnel through each other, a part of their wavefunction is trapped inside the other soliton; this effect grows as the interspecies interaction increases leading to a slow increase in the standard deviation of the atomic density distributions.

A rather sudden change takes place at about $\Lambda_{1,2}=6$. The system becomes visibly sensitive to small changes in the mutual interaction. This is the regime where the collision results in the destruction of the two breathers. Fig. 5 is an example of such a case. The process is chaotic, yet in many cases leads to the creation of a double-species symbiotic breather manifested by its very low FWHM.

\section{A. Discussion}

The presented results can be reasonably explained by using one of the key phenomena at the base of gap solitons: the negative effective mass. The dynamics of a gap soliton in an external potential are exactly opposite to what one would expect - a gap soliton attempts to climb potential hills and in itself is a balance between its negative effective mass that tries to make it collapse and its repulsive self-interaction that prevents it $[11,27]$. In fact, the variational model of a wavepacket used in Trombettoni et al. [11] shows that the wavepacket center obeys a Newton-like dynamics when $p \approx 0$ and exactly the contrary when $p \approx \pi$.

This 'contrary' behavior of the solitons seems to be the key to the explanation of our findings. The totally elastic collision encountered when the interspecies interaction is highly attractive would be caused by the fact that the solitons 'see' each other as potential walls rather than wells.

The splitting behavior has been investigated in a slightly different context by Matuszewski et al. [27] where the dynamics of two already overlapped stationary solitons was analyzed. In our case splitting happens if the attractive interaction is small enough to let the two breathers overlap briefly. Then the system becomes unstable and each breather splits in two. It is also possible to look at this phenomenon from a different angle. Due to its negative effective mass, the split of the breather is quite similar to the case of a wavepacket encountering a potential barrier where, depending on the barrier height (or the interaction between the breathers) part of the wavepacket goes through while the rest is reflected.

In the repulsive interaction regime the breathers behave as if they saw each other as potential wells. This is again an effect of their negative effective mass and, consequently, reversed dynamics. Thus, for the collision's duration, their speed increases (at the cost of wavepacket spreading and of a reduction of their energy due to atomic self-interaction).

The chaotic behavior when the interspecies interaction is large and repulsive is probably caused by the system entering an unstable regime as predicted by Gubeskys et al.[24]. The chaotic dynamics would then cause the destruction of the original two breathers and possibly the creation of a stable intragap soliton. It is beyond the capabilities of our model to establish if it is possible for an intergap soliton to emerge during the collision since the tight-binding approximation is limited to the lowest band-gap by definition.

\section{CONCLUSION}

We have analyzed the behavior of interacting and colliding discrete breathers in BEC composed of different atomic species in optical lattices. We have found that the interaction depends on the initial distance of the two breathers and led either to the formation of a symbiotic solitons or to the setup in motion of one of the two breathers. The collision outcome depends both on the tunneling rate ratios of the two species, as well as the interspecies interactions. When the tunneling rates differ greatly, as in the ${ }^{41} \mathrm{~K}+{ }^{87} \mathrm{Rb}$ mixture, one of the breathers 
acts as an effective potential wall to the other and the whole process can be viewed as a case of one-particle scattering on a potential wall.

In the case where the tunneling rates are comparable (like in the case of mixtures of ytterbium isotopes) we have identified four collision regimes. For large negative scattering lengths the collision is elastic and the two traveling breathers remain intact, with considerable momentum transfer between the two. For small negative scattering rates, the breathers overlap briefly and split in two, as originally predicted in [27]. When the interspecies interaction is weakly repulsive, the two breathers tunnel through each other unharmed for a wide range of interspecies interacions. Finally, with the interspecies interaction sufficiently large, the dynamics becomes chaotic and the two breathers are destroyed with a possible creation a new two-component soliton similar to an intragap soliton as predicted in [24]. Feasible explanations to the above phenomena have been provided using the concept of negative effective mass and the resulting reversed dynamics.

Interaction and collision properties of localized excitations in BEC in optical lattices can have interesting applications in the realization of ultracold Bose-Fermi mixtures where gap solitons can be viewed as matter-wave counterparts of quantum dots and antidots [64]. Changing the species interaction allows one to tune the character of the collisions from fully elastic to fully inelastic and/or tunneling with clear advantages in the manipulation of information in matter-wave systems.

\section{Acknowledgements}

We are indebted to Stefano Iubini, Massimo Inguscio, Francesco Minardi, Roberto Franzosi and Yoshiro Takahashi for useful discussions. This work was partially supported by the Institute of Complex Systems at Strathclyde and an EPSRC doctoral training fellowship. The research is part of the program of the National Laboratory FAMO in Torun, Poland and partially supported by the Polish MNISW (Project No. N N202 1489 33).

\section{Appendix: Simulations of the Continuous Model}

The DNLS model describes BEC in optical lattices in the limit of deep potential wells. The interactions and collision mechanisms of breathers in two-species BECs presented in Sections III, IV and V survive the tight-binding approximation. Here we use a scaled version of the one-dimensional Gross-Pitaevskii equation [65] extended to two-species BEC in optical lattices:

$$
i \dot{u}_{i}=\left(-\frac{\partial^{2}}{\partial x^{2}}+V_{0} \sin ^{2}\left(\frac{\pi x}{2}\right)+\sum_{j=1,2} \beta_{i, j}\left|u_{j}\right|^{2}\right) u_{i},
$$

where $u_{i}$ is the wavefunction of the $i$-th species, $V_{0}=10$ is the potential depth scaled to the recoil energy, $\beta_{i, i}$ and $\beta_{1,2}=\beta_{2,1}$ are the intra- and interspecies interaction parameters respectively. The spatial variable $x$ is now continuous and the Lapla- (a)

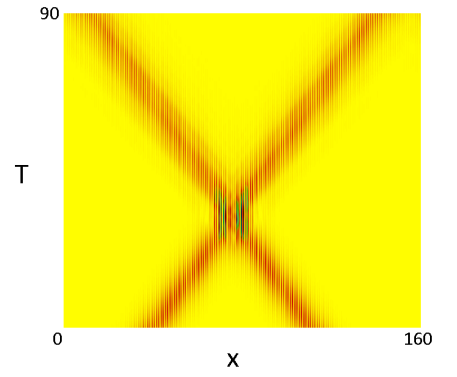

(b)

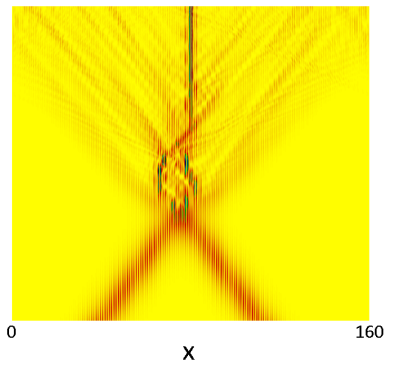

FIG. 8: (Color online) Collision of two traveling breathers in the continuous case (A.1) for $\beta_{i, i}=1$. $\beta_{1,2}$ is set to (a) -18 , giving an elastic collision, and (b) 20.4, with the destruction of the breathers and creation of a symbiotic one. These results are similar to those displayed in Fig. 3(b) and (c) for the DNLS model.

cian term describes the momentum of the atoms in the lattice. We show here that the collision mechanisms survive from the discrete to continuous limit by simulating an optical lattice of 80 potential wells.

In Fig. 8 (a), we show an elastic collision of moving lattice solitons (continuous breathers) with a large attractive interspecies interaction of $\beta_{i, j}=-18$ obtined by the numerical integration of Eq. (A.1). Likewise, inelastic collisions take place with repulsive interactions as shown in Fig. 8, with $\beta_{i, j}=20.4$. The inelastic collision here ends in the formation of a symbiotic lattice soliton.

The simulations of the continuous model (A.1) take at least three times as long as those of the discrete model, even with the smaller number of potential wells ( 80 in the continuous compared to 127 in the discrete). We also note that the numerical method used for the continuous case is not symplectic and uses periodic boundary conditions that limit its application. In spite of these limitations, the results of the continuous model simulations confirm those of the DNLS model in the deep potential case as demonstrated in Fig. 8. For these reasons, the DNLS model represent an accurate and trustworthy testbench for the realistic investigation of the collision mechanism of breathers in two-species BEC as shown for example in the exhaustive Fig. 7.
[1] F. Dalfovo, S. Giorgini, L. P. Pitaevskii, and S. Stringari, Rev. Mod. Phys. 71, 463 (1999).
[2] L. P. Pitaevskii and S. Stringari, Bose-Einstein Condensation, (Clarendon Press, Oxford, 2004) 
[3] O. Morsch and M. Oberthaler, Rev. Mod. Phys. 78, 179 (2006)

[4] I. Bloch, J. Dalibard, and W. Zwerger, Rev. Mod. Phys. 80, 885 (2008)

[5] M. Greiner, O. Mandel, T. Esslinger, T. W. Hänsch, and I. Bloch, Nature 415, 39 (2002)

[6] M. L. Chiofalo and M. P. Tosi, J. Phys. B 34, 4551 (2001)

[7] G. Roati, C. D'Errico, L. Fallani, M. Fattori, C. Fort, M. Zaccanti, G. Modugno, M. Modugno and M. Inguscio, Nature 453, 895 (2008)

[8] C. J. Pethick and H. Smith, Bose-Einstein Condensation in Dilute Gases (Cambridge University Press, Cambridge, 2013)

[9] R. Franzosi, R. Livi, G.-L.Oppo, and A. Politi, Nonlinearity 24, R89 (2011)

[10] K. E. Strecker, G. B. Partridge, A. G. Truscott, and R. G. Hulet, Nature 417, 6885 (2002); D. J. Frantzeskakis, J. Phys. A 23, 213001 (2010)

[11] A. Trombettoni and A. Smerzi, Phys. Rev. Lett. 86, 002353 (2001).

[12] F. Kh. Abdullaev, B. B. Baizakov, S. A. Darmanyan, V. V. Konotop, and M. Salerno, Phys. Rev. A 64, 043606 (2001)

[13] H. A. Cruz, V. A. Brazhnyi, V. V. Konotop, and M. Salerno, Physica D 238, 1372 (2009)

[14] B. Eiermann, P. Treutlein, Th. Anker, M. Albiez, M. Taglieber, K.-P. Marzlin, and M. K. Oberthaler, Phys. Rev. Lett. 91, 060402 (2003); Th. Anker, M. Albiez, R. Gati, S. Hunsmann, B. Eiermann, A. Trombettoni, and M. K. Oberthaler, Phys. Rev. Lett 94, 020403 (2005)

[15] M. Matuszewski, W. Krolikowski, M. Trippenbach, and Y. S. Kivshar, Phys. Rev. A 73, 063621 (2006)

[16] R. Livi, R. Franzosi, and G.-L. Oppo, Phys. Rev. Lett. 97, 060401 (2006)

[17] R. Franzosi, R. Livi, and G.-L. Oppo, J. Phys. B 401195 (2007)

[18] D. S. Hall, M. R. Matthews, J. R. Ensher, C. E. Wieman, and E. A. Cornell, Phys. Rev. Lett. 81, 1539 (1998)

[19] P. Öhberg and S. Stenholm, Phys. Rev. A 57, 1272 (1998)

[20] S. B. Papp, J. M. Pino, and C. E. Wieman, Phys. Rev. Lett. 101, 040402 (2008)

[21] S. Ronen, J. L. Bohn, L. E. Halmo, and M. Edwards, Phys. Rev. A 78, 053613 (2008)

[22] S. Hooley and K. A. Benedict, Phys. Rev. A 75, 033621 (2007)

[23] J. Ruostekoski and Z. Dutton, Phys. Rev. A 76, 063607 (2007)

[24] A. Gubeskys, B. A. Malomed, and I. M. Merhasin, Phys. Rev. A 73, 023607 (2006)

[25] S. K. Adhikari and B. A. Malomed, Phys. Rev. A, 77, 023607 (2008)

[26] B. A. Malomed, D. J. Kaup, and R. A. Van Gorder, Phys. Rev. E 85, 026604 (2012)

[27] M. Matuszewski, B. A. Malomed, and M. Trippenbach, Phys. Rev. A 76, 043826 (2007)

[28] Sk. Golam Ali and B. Talukdar, Annals of Physics 324, 1194 (2009)

[29] H. A. Cruz, V. A. Brazhnyi, V. V. Konotop, G. L. Alfimov, and M. Salerno, Phys. Rev. A 76, 013603 (2007)

[30] F. Kh. Abdullaev, A. Gammal, M. Salerno, and L. Tomio, Phys. Rev. A, 77, 023615 (2008).

[31] Z. Shi, K. J. H. Law, P. G. Kevrekidis, and B. A. Malomed, Phys. Lett. A 372, 4021 (2008)

[32] S. K. Adhikari, J. Phys. B 44, 075301 (2011)

[33] A. I. Yakimneko, K. O. Shchebetovska, S. I. Vilchinskii, and M. Weyrauch, Phys. Rev. A 85, 053640 (2012)

[34] Z. M. He, D. L. Wang, J. W. Ding, and X. H. Yan, Eur. Phys. J. D 66, 139 (2012)

[35] V. M. Perez-Garcia and J. B. Beita, Phys. Rev. A. 72, 033620
(2005)

[36] B. J Dabrowska, E. A. Ostrovskaya and Y. S. Kivshar, J. Opt. B: Quantum Semiclass. Opt. 6423 (2004)

[37] X. F. Zhang, X. H. Hu, X. X. Liu, and W. M. Liu, Phys. Rev. A 79, 033630 (2009)

[38] Th. Busch and J. R. Anglin, Phys. Rev. Lett. 87, 010401 (2001)

[39] Y. Takasu, K. Maki, K. Komori, T. Takano, K. Honda, M. Kumakura, T. Yabuzaki, and Y. Takahashi, Phys. Rev. Lett. 91, 040404 (2003)

[40] T. Fukuhara, S. Sugawa, and Y. Takahashi, Phys. Rev. A 76, 051604(R) (2007)

[41] S. Sugawa, R. Yamazaki, S. Taie, and Y. Takahashi, Phys. Rev. A 84, 011610(R) (2007)

[42] T. Fukuhara, S. Sugawa, Y. Takasu, and Y. Takahashi, Phys. Rev. A 79, 021601(R) (2009)

[43] K. Kasamatsu and M. Tsubota, J. Low Temp. Phys. 150, 599 (2008).

[44] K. Enomoto, M. Kitagawa, K. Kasa, S. Tojo, and Y. Takahashi, Phys. Rev. Lett. 98, 203201 (2007)

[45] M. Kitagawa, K. Enomoto, K. Kasa, Y. Takahashi, R. Ciurylo, P. Naidon, and P. S. Julienne, Phys. Rev. A 77, 012719 (2008)

[46] R. Ciurylo, E. Tiesinga, and P. S. Julienne, Phys. Rev. A 71, 030701(R) (2005)

[47] K. Enomoto, K. Kasa, M. Kitagawa, and Y. Takahashi, Phys. Rev. Lett. 101, 203201 (2008)

[48] M. Borkowski, R. Ciurylo, P. S. Julienne, S. Tojo, K. Enomoto, and Y. Takahashi, Phys. Rev. A 80, 012715 (2009)

[49] M. Borkowski, R. Ciurylo, P. S. Julienne, R. Yamazaki, H. Hara, K. Enomoto, S. Taie, S. Sugawa, Y. Takasu, and Y. Takahashi , Phys. Rev. A 84, 030702 (2011)

[50] G. Thalhammer, G. Barontini, L. De Sarlo, J. Catani, F. Minardi, and M. Inguscio, Phys. Rev. Lett 100, 210402 (2008)

[51] T. Neff, H. Hennig, and R. Fleishmann, Dynamical phase diagram of Gaussian BEC wave packets in optical lattices arXiv:1309.7939 (2014)

[52] W. Kohn, Phys. Rev. 115, 809 (1959)

[53] H. Yoshida, Phys. Lett. A 170, 262 (1990)

[54] S. Iubini, R. Franzosi, R. Livi, G.-L.Oppo, and A. Politi, New Journal of Physics 15, 023032 (2013)

[55] M. Inguscio, private communication.

[56] A. Marte, T. Volz, J. Schuster, S. Dürr, G. Rempe, E. G. M. van Kempen, and B. J. Verhaar, Phys. Rev. Lett. 89, 283202 (2002)

[57] G. Modugno, M. Modugno, F. Riboli, G. Roati, and M. Inguscio, Phys. Rev. Lett. 89, 190404 (2002)

[58] H. Wang, A. N. Nikolov, J. R. Ensher, P. L. Gould, E. E. Eyler, W. C. Stwalley, J. P. Burke, Jr., J. L. Bohn, C. H. Greene, E. Tiesinga, C. J. Williams, and P. S. Julienne, Phys. Rev. A 62, 052704 (2000)

[59] J. Catani, L. De Sarlo, G. Barontini, F. Minardi, and M. Inguscio, Phys. Rev. A 77, 011603(R) (2008)

[60] J. Gomez-Gardenes, L. M. Floria, M. Peyrard, and A. R. Bishop, Chaos 14, 1130 (2004)

[61] L. Fallani, F. S. Cataliotti, J. Catani, C. Fort, M. Modugno, M. Zawada, and M. Inguscio, Phys. Rev. Lett. 91, 240405 (2003)

[62] S. Flach and A. V. Gorbach, Phys. Rep. 467, 1 (2008)

[63] Simulations showing traveling breathers bouncing at boundaries are presented in [9]. Details about the mechanism behind this phenomenon will be presented elsewhere.

[64] M. Salerno, Phys. Rev. A 72, 063602 (2005)

[65] N. K. Efremidis and D. N. Christodoulides, Phys. Rev. A 67, 063608 (2003) 